Article

\title{
Raman 2D Peak Line Shape in Epigraphene on SiC
}

\author{
Jan Kunc *(i) and Martin Rejhon \\ Institute of Physics, Faculty of Mathematics and Physics, Charles University, Ke Karlovu 5, \\ CZ-121 16 Prague 2, Czech Republic \\ * Correspondence: kunc@karlov.mff.cuni.cz
}

Received: 29 February 2020; Accepted: 23 March 2020; Published: 30 March 2020

check for updates

\begin{abstract}
We measured a 2D peak line shape of epitaxial graphene grown on $\mathrm{SiC}$ in high vacuum, argon and graphene prepared by hydrogen intercalation from the so called buffer layer on a silicon face of $\mathrm{SiC}$. We fitted the 2D peaks by Lorentzian and Voigt line shapes. The detailed analysis revealed that the Voigt line shape describes the 2D peak line shape better. We have determined the contribution of the homogeneous and inhomogeneous broadening. The homogeneous broadening is attributed to the intrinsic lifetime. Although the inhomogeneous broadening can be attributed to the spatial variations of the charge density, strain and overgrown graphene ribbons on the sub-micrometer length scales, we found dominant contribution of the strain fluctuations. The quasi free-standing graphene grown by hydrogen intercalation is shown to have the narrowest linewidth due to both homogeneous and inhomogeneous broadening.
\end{abstract}

Keywords: epitaxial graphene; silicon carbide; Raman spectroscopy; 2D peak line shape; G peak; charge density; strain

\section{Introduction}

The Raman spectroscopy of graphene is a well-established technique [1-3] to determine number of graphene layers [4], strain [5,6], charge density [6-9], grain size [10-14], graphene functionalization [15], misorientation of graphene layers [16] or degree of hydrogen intercalation of epitaxial graphene on $\mathrm{SiC}[17,18]$. The graphene's most prominent Raman spectral features are the $\mathrm{D}$ peak, $\mathrm{G}$ peak and 2D peak. The $D$ peak reflects the amount of defects, or the graphene grain size. The $G$ peak is related to the in-plane bond-stretching optical vibrations of $\mathrm{sp}^{2}$ hybridized carbon atoms in the graphene lattice $[19,20]$. The 2D peak is recognized as a combination mode of lattice and electronic excitations. The predicted unique property of the $2 \mathrm{D}$ peak is its line shape. The $2 \mathrm{D}$ peak is predicted to have a Lorentzian line shape in the case of the single layer graphene (SLG) [21]. The 2D peak is predicted to have four components in the case of bilayer graphene. The single and four-component nature of the $2 \mathrm{D}$ peak was proved experimentally [22,23]. It is also known that the spectral position of the 2D peak is determined by the uniaxial $[24-27]$ and biaxial $[5,6,26]$ mechanical strain and charge density $[7,8]$ of the graphene layer. The scaling of the 2D peak position with charge and strain was studied extensively. The 2D peak line shape is influenced by the strain uniformity [28] and strain fluctuations on the nanometer scale [29]. Also, different line shape was identified for graphene on SiC terraces and step edges [30].

However, beside the known spectral line shape and parameters determining the position of the 2D peak, there is little known about the combined effect of the homogeneous and inhomogeneous broadening. The knowledge of the mutual effects of the homogeneous and inhomogeneous broadening can provide deeper insight into the formation of the graphene layers and it can be used to further optimize graphene growth. Hence, we propose here to analyze in detail the spectral line shape of the 2D peak. Instead of describing the 2D peak line shape only by the Lorentzian broadening, we assume 
also the contribution by the inhomogeneous broadening. The homogeneous and inhomogeneous broadening have to be taken into account simultaneously. As the inhomogeneous broadening describes the random nature of the parameters determining the position of 2D peak, it is described by the normal (Gaussian) distribution. The mutual effect of the homogeneous and inhomogeneous broadening thus leads to the convolution of the Lorentzian and Gaussian broadening, also called the Voigt broadening. We show here that the Voigt profile describes the 2D peak line shape better than the Lorentzian profile. We show that the Voigt broadening, though it has one more fitting parameter, it does not show any signatures of over-parametrized model. We test the better fit quality by the F-test and the results are compared for three different samples and four different locations on each sample.

\section{Materials and Methods}

The epitaxial graphene was grown on a Si-face of $6 \mathrm{H}-\mathrm{SiC}$ by thermal decomposition [31-34]. We grew three samples, each under different growth conditions. The sample grown in vacuum $[31,32]$ at $10^{-5} \mathrm{mbar}$ was heated to $1600{ }^{\circ} \mathrm{C}$ for $5 \mathrm{~min}$. The sample grown in argon [33] at $1050 \mathrm{mbar}$ was heated at $1650^{\circ} \mathrm{C}$ for $5 \mathrm{~min}$. The quasi free-standing monolayer graphene (QFMLG) [35,36] was grown in two steps. First, the graphene buffer layer was grown in 1050 mbar of argon at $1550{ }^{\circ} \mathrm{C}$ for $5 \mathrm{~min}$. The second growth step was the hydrogen intercalation at $1120^{\circ} \mathrm{C}$ for $5 \mathrm{~min}$ followed by a 2 hours long cooling to $600{ }^{\circ} \mathrm{C}$. The growth temperature was adjusted with respect to the sublimation rate of silicon from the heated $\mathrm{SiC}$ wafer. The growth in high vacuum allows high silicon sublimation rates, hence, the temperature is reduced to $1600{ }^{\circ} \mathrm{C}$ in contrast to growth in 1050 mbar of argon, where the growth temperature has to be increased by $50{ }^{\circ} \mathrm{C}$ to $1650{ }^{\circ} \mathrm{C}$ to promote silicon sublimation comparable to the sublimation rate in high vacuum. The growth of QFMLG requires to grow so called buffer layer first. The buffer layer grows at even lower temperature than the single layer graphene. For this reason, the first growth step is performed at $1050 \mathrm{mbar}$ of argon at $1550{ }^{\circ} \mathrm{C}$ for $5 \mathrm{~min}$. The buffer layer is the graphene lattice, where about $30 \%$ carbons are $\mathrm{sp}^{3}$ bonded to the underlying $\mathrm{SiC}$ substrate [37]. This $\mathrm{sp}^{3}$ bonding was switched into $\mathrm{sp}^{2}$ bonding by hydrogen intercalation $[17,38]$. More details on graphene growth and hydrogen intercalation can be found in our previous works $[17,18,39]$.

Raman spectra were measured by WITec alpha300 (WITec, Ulm, Germany) micro-Raman confocal microscope. The Raman spectra were excited by $532 \mathrm{~nm}$ laser light. We used $25 \mathrm{~mW}$ laser power and the laser spot diameter was $1 \mu \mathrm{m}$ in the focal plane. The spectra were acquired in two $30 \mathrm{~s}$ accumulations to achieve low level of noise in the tails of the 2D peak. The spatial Raman maps were measured on the area $4 \times 4 \mu \mathrm{m}^{2}$ and we accumulated Raman spectra twice $5 \mathrm{~s}$ to optimize lateral resolution, noise level and the total measurement time. The Atomic Force Microscopy (AFM) and Lateral Force Microscopy (LFM) were measured by WITec alpha300 (WITec, Ulm, Germany) AFM. We measured AFM and LFM in the contact mode, and, the scanned area was $20 \times 20 \mu^{2}$.

The 2D peak was fitted by Lorentzian and Voigt line shapes. The Lorentzian line shape was taken in the form of Equation (1)

$$
G_{L}=\frac{\gamma}{\pi\left[\left(x-x_{0}\right)^{2}+\gamma^{2}\right]}
$$

where $x_{0}$ is the spectral position of the peak, $\gamma$ determines the width of the 2D peak. The Equation (1) is scaled by a factor $I_{0}$ describing the intensity of the Lorentzian peak. Parameter $\gamma$ is related to the more experimentally accessible Full Width at Half Maximum (FWHM) of the Lorentzian peak $f_{L}$ by Equation (2).

$$
f_{L}=2 \gamma
$$

The Voigt line shape is given by the convolution of the Lorentzian Equation (1) and Gaussian Equation (3) line shape

$$
G_{G}=\frac{1}{\sqrt{2 \pi} \sigma} e^{-\frac{\left(x-x_{0}\right)^{2}}{2 \sigma^{2}}}
$$


The Gaussian broadening $\sigma$ relates to the $\mathrm{FWHM} f_{G}$ by Equation (4)

$$
f_{G}=2 \sigma \sqrt{2 \ln (2)}
$$

As the convolution of the Gaussian and Lorentzian function is numerically demanding, we used a common approximation of the Voigt line shape [40,41] given by Equation (5)

$$
G_{V}=I_{0}\left[\eta G_{L}+(1-\eta) G_{G}\right]
$$

where $\eta$ is a function of the total $\operatorname{FWHM} f, f_{G}$ and $f_{L}$

$$
\eta=1.36603 \frac{f_{L}}{f}-0.47719\left(\frac{f_{L}}{f}\right)^{2}+0.11116\left(\frac{f_{L}}{f}\right)^{3} .
$$

The total Voigt broadening $f$ is given by Equation (7)

$$
f=\left(f_{G}^{5}+2.69269 f_{G}^{4} f_{L}+2.42843 f_{G}^{3} f_{L}^{2}+4.47163 f_{G}^{2} f_{L}^{3}+0.07842 f_{G} f_{L}^{4}+f_{L}^{5}\right)^{1 / 5} .
$$

\section{Results}

We show in Figure 1 the typical Raman spectra of graphene grown in high vacuum, argon and the intercalated buffer, so called QFMLG. All spectra show the typical graphene characteristics as the D peak, G peak and 2D peak. The Raman spectra also show typical characteristics of the single-layer graphene. These are the ratio of the integrated 2D peak to $G$ peak intensity larger than 2 , and, the single-component line shape of the 2D peak. The characteristic distinction between the hydrogen intercalated and non-intercalated samples can be also found by the absence/presence of the peaks labeled (1), (2) and (3) in inset of Figure 1. These peaks were attributed to the buffer layer [18,42]. The hydrogenated (QFMLG) and non-hydrogenated (SLG-vac, SLG-Ar) samples can be also distinguished by a low intensity background in the spectral range of the $\mathrm{D}$ peak. This background was assigned to the buffer layer, too [42]. We observe rather similar Raman spectra of graphene grown in argon and high vacuum. Their differences are discussed in the following detailed analysis.

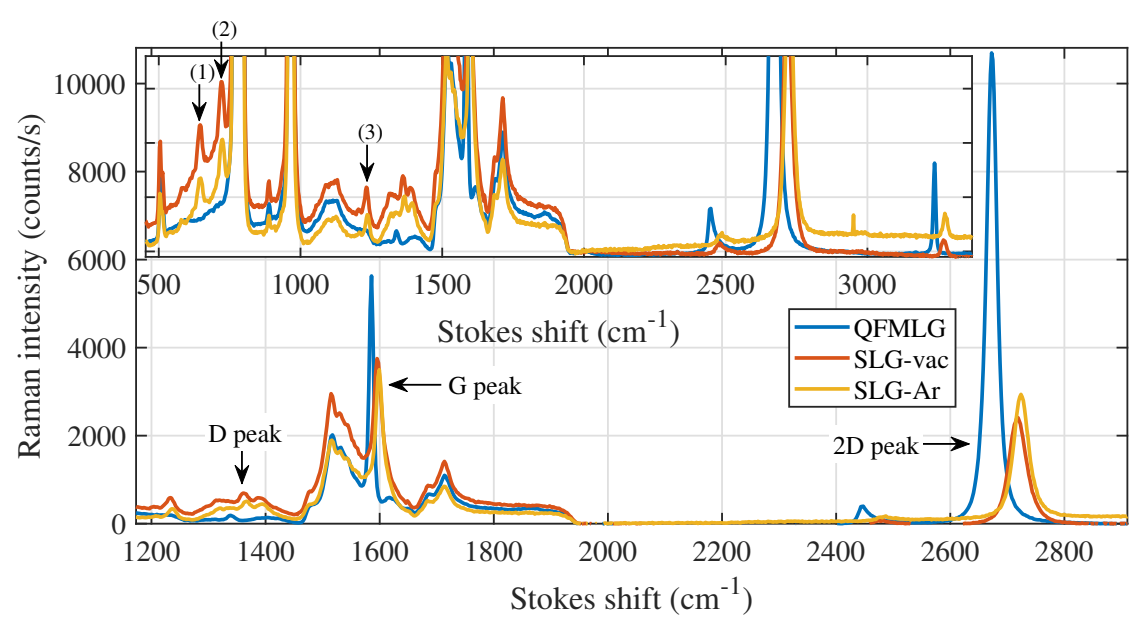

Figure 1. Raman spectra of quasi free-standing monolayer graphene (QFMLG), single layer graphene grown in vacuum (SLG-vac) and single layer graphene grown in argon (SLG-Ar). Graphene related D, $\mathrm{G}$ and 2D peaks are labeled by arrows. Inset shows details of Raman spectra including three buffer related Raman modes, labeled by arrows (1), (2) and (3).

The 2D peaks of the three samples are shown in Figure 2 by black circles. We obtained the spectra by two $30 \mathrm{~s}$ integration periods. The $30 \mathrm{~s}$ integration reduced the noise level and the two accumulations allowed us to remove spikes in recorded spectra. The low level of the noise is essential to fit the 2D 
peak line shape at low-energy and high-energy tails from the central peak position. Before we analyzed the $2 \mathrm{D}$ peak line shape, we subtracted a linear background. The background was determined using five experimental points at 2530 and at $2870 \mathrm{~cm}^{-1}$. The 2D peaks are fitted by the Lorentzian (green curves) and Voigt (red curves) line shapes in Figure 2. The residuals are displayed in the top insets and the corresponding histograms of the residuals are shown in the bottom insets of Figure 2.
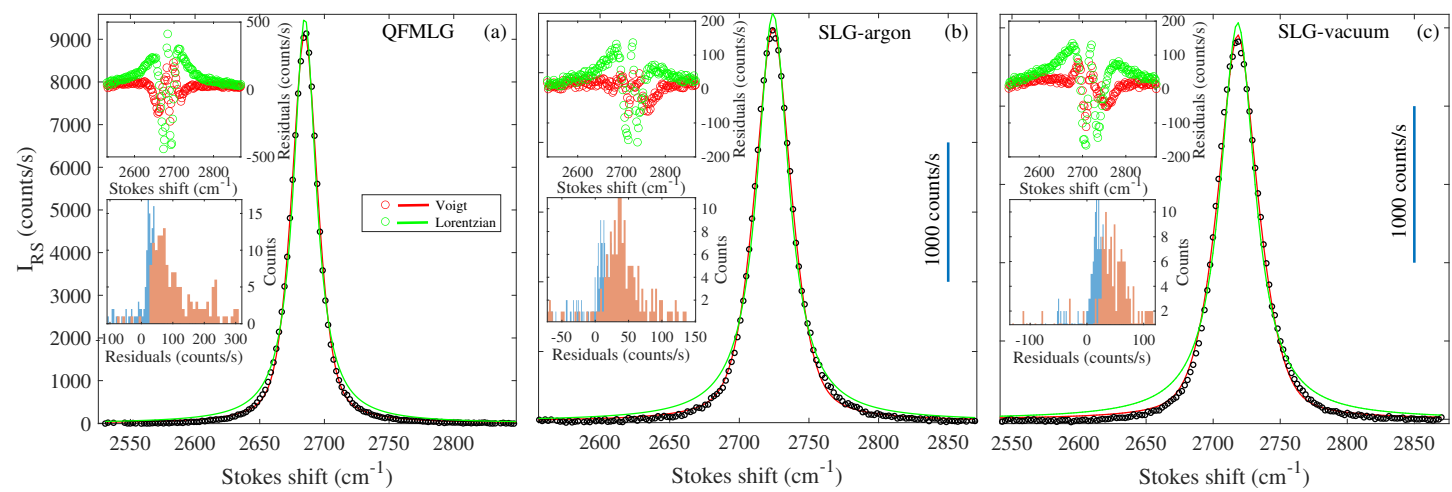

Figure 2. Line shape of the 2D peak in (a) QFMLG, (b) SLG-Ar and (c) SLG-vac. The black dots are the experimental data, green curve is a Lorentzian fit and red curve is the Voigt fit to the experimental data. The upper insets show the residuals for the (green point) Lorentzian and (red points) Voigt line shape. The bottom insets show the histograms of thee residuals.

The fit improvement by the Voigt line shape is tested by the F-test. The Degrees of Freedom (DOF) and Residual Sum of Squares of the Lorentzian (model 1) and Voigt (model 2) line shape are summarized in Table 1. The null hypothesis for the F-test is: the Lorentzian and Voigt line shapes are the same. The F-number in Table 1 results from the comparison of the two models. The p-number determines the cumulative probability that the expected F number is lower than the experimentally determined F-number.

Table 1. Parameters used to perform the F-test. Degrees of freedom (DOF), Residual Sum of Squares $\left(\mathrm{RSS}_{1}\right)$ of the Lorentzian model and RSS 2 of the Voigt model. $\mathrm{F}$ is the value of the F-statistics. Number $p$ is the p-number to reject the null hypothesis.

\begin{tabular}{lccccc}
\hline Sample & DOF & RSS $_{1}$ & RSS $_{2}$ & F & p \\
\hline QFMLG & 163 & $3.6 \times 10^{6}$ & $4.9 \times 10^{5}$ & 1000 & 1 \\
SLG-Ar & 162 & $5.8 \times 10^{5}$ & $8.4 \times 10^{4}$ & 932 & 1 \\
SLG-vac & 163 & $8.0 \times 10^{5}$ & $1.5 \times 10^{5}$ & 675 & 1 \\
\hline
\end{tabular}

The F-test shows that the Voigt line shape describes the 2D peak line shape better. As the Voigt line shape has four fitting parameters (one more with respect to the Lorentzian broadening), we need to verify that the Voigt line shape model is not overparametrized. We verify the overparametrization by determining the $\chi^{2}$ statistics of the fitted models. The $\chi^{2}$ statistics requires the experimental error $\sigma_{\text {exp }}$. We determine the experimental error from the high-energy tails of the 2D peak, as depicted in Figure 3. The high-energy tail is fitted by the second order polynomial to describe the trend of the experimental data. The residuals are considered as a random experimental error. The normality of these residuals is tested by the Kolmogorov-Smirnov test, as depicted by the cumulative distribution function in the inset of Figure 3. The normality is verified at the $95 \%$ confidence level. The experimental error is estimated to be $\sigma_{\text {exp }} \approx 10 \mathrm{~cm}^{-1}$. The centered and normalized $\chi^{2}$ for the Lorentzian and Voigt model is 1000 and 260 , respectively. 


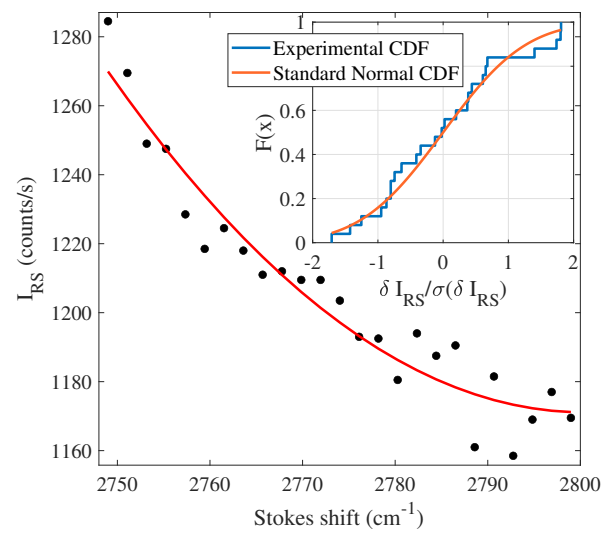

Figure 3. The high-energy tail of the 2D peak (black points) was fitted to the polynomial of the 2nd order (red curve) to determine the experimental error. The normality of the residuals is tested by the Kolmogorov-Smirnov test (inset).

We fitted the 2D peak spectra at four different positions on each sample. The statistics for the three samples is shown in Figure 4 and summarized in Table 2. The FWHM was obtained directly from the experimental data without using any fitting procedure. The parameters $\gamma$ and $\sigma$ are the fitting parameters of the Voigt line shape. We observe that the QFMLG shows the narrowest broadening. The QFMLG also shows the smallest contribution of the homogeneous and inhomogeneous broadening. It can be seen in Figure 4 that the homogeneous and inhomogeneous broadening are similar in QFMLG. However, the inhomogeneous broadening is larger by $2-3 \mathrm{~cm}^{-1}$ with respect to the homogeneous broadening in SLG-Ar and SLG-vac. The role of inhomogeneous broadening is thus larger in SLG-Ar and SLG-vac than in the QFMLG.
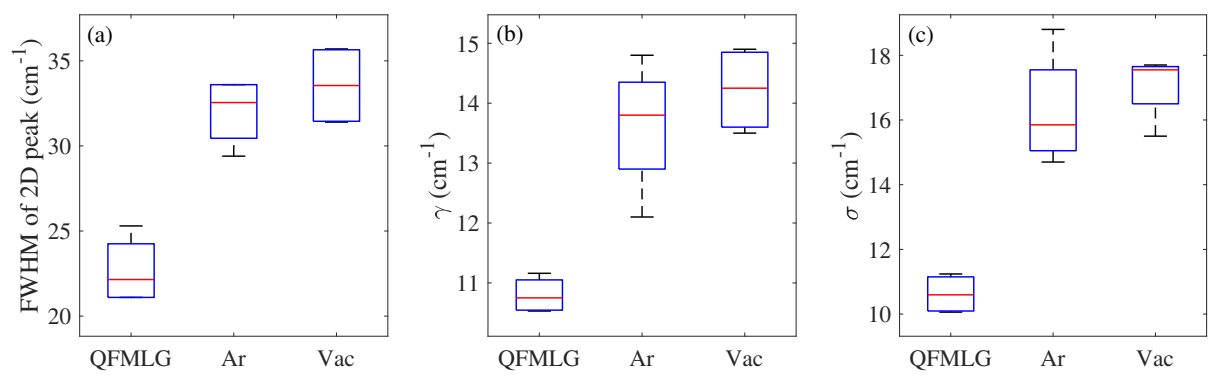

Figure 4. The (a) FWHM and (b,c) Voigt fitting parameters of (b) homogeneous $(\gamma)$ and (c) inhomogeneous $(\sigma)$ broadening are compared for the three samples (QFMLG, SLG-Ar, SLG-vac).

Table 2. Fitted parameters $\gamma$ and $\sigma$ of Voigt line shape and FWHMs for three samples (QFMLG, SLG-Ar, SLG-vac). The line shape was determined at four different positions on each sample.

\begin{tabular}{ccccc}
\hline Sample & Sample Position & FWHM $\left(\mathbf{c m}^{-\mathbf{1}}\right)$ & $\gamma\left(\mathbf{c m}^{-\mathbf{1}}\right)$ & $\sigma\left(\mathbf{c m}^{-\mathbf{1}}\right)$ \\
\hline QFMLG & 1 & 21.1 & $10.53 \pm 0.12$ & $10.13 \pm 0.12$ \\
& 2 & 21.1 & $10.56 \pm 0.13$ & $10.06 \pm 0.13$ \\
& 3 & 25.3 & $11.16 \pm 0.16$ & $11.24 \pm 0.15$ \\
Argon grown & 4 & 23.2 & $10.94 \pm 0.17$ & $11.06 \pm 0.16$ \\
\hline \multirow{2}{*}{ Vacuum grown } & 1 & 33.6 & $14.8 \pm 0.4$ & $16.3 \pm 0.3$ \\
& 3 & 31.5 & $13.7 \pm 0.3$ & $15.4 \pm 0.3$ \\
& 4 & 29.4 & $13.9 \pm 0.2$ & $14.7 \pm 0.2$ \\
& 2 & 33.6 & $12.1 \pm 0.6$ & $17.6 \pm 0.5$ \\
\hline & 3 & 35.7 & $14.9 \pm 0.5$ & $17.5 \pm 0.4$ \\
& 4 & 31.5 & $13.5 \pm 0.3$ & $15.5 \pm 0.2$ \\
& & 35.6 & $14.8 \pm 0.4$ & $17.7 \pm 0.4$ \\
& & 31.4 & $13.7 \pm 0.3$ & $16.4 \pm 0.2$ \\
\hline
\end{tabular}


We study sources of inhomogeneous broadening by measuring spatial Raman maps. We show the Raman maps of the 2D peak position and FWHM of 2D peak in Figure 5. We select three representative points (marked by black, red and blue circles in Figure 5a-f) and Raman spectra taken at these points are plotted in Figure 5g-i. The SLG-vac sample is the least homogeneous. The broad and blue-shifted 2D peak (red spectrum in Figure 5g) is a fingerprint of bilayer graphene. We observe similar small areas of broad and blue-shifted 2D peak in QFMLG, too (red spectrum in Figure 5i). Though SLG-Ar sample appears to be more homogeneous than SLG-vac and QFMLG, this could be due to the larger steps of $\mathrm{SiC}$, or, due to the specific area chosen for the Raman map. To verify further the origin of these inhomogeneities, we measured also AFM and LFM, see Figure 6. The topography of SLG-vac shows circular-like $\mathrm{SiC}$ terraces of 1-4 $\mu \mathrm{m}$ in diameter. The SLG-Ar and QFMLG show regular SiC step bunching. The regular terraces are 1-4 $\mu \mathrm{m}$ broad in SLG-Ar, and, they are 6-7 $\mu \mathrm{m}$ broad in QFMLG.

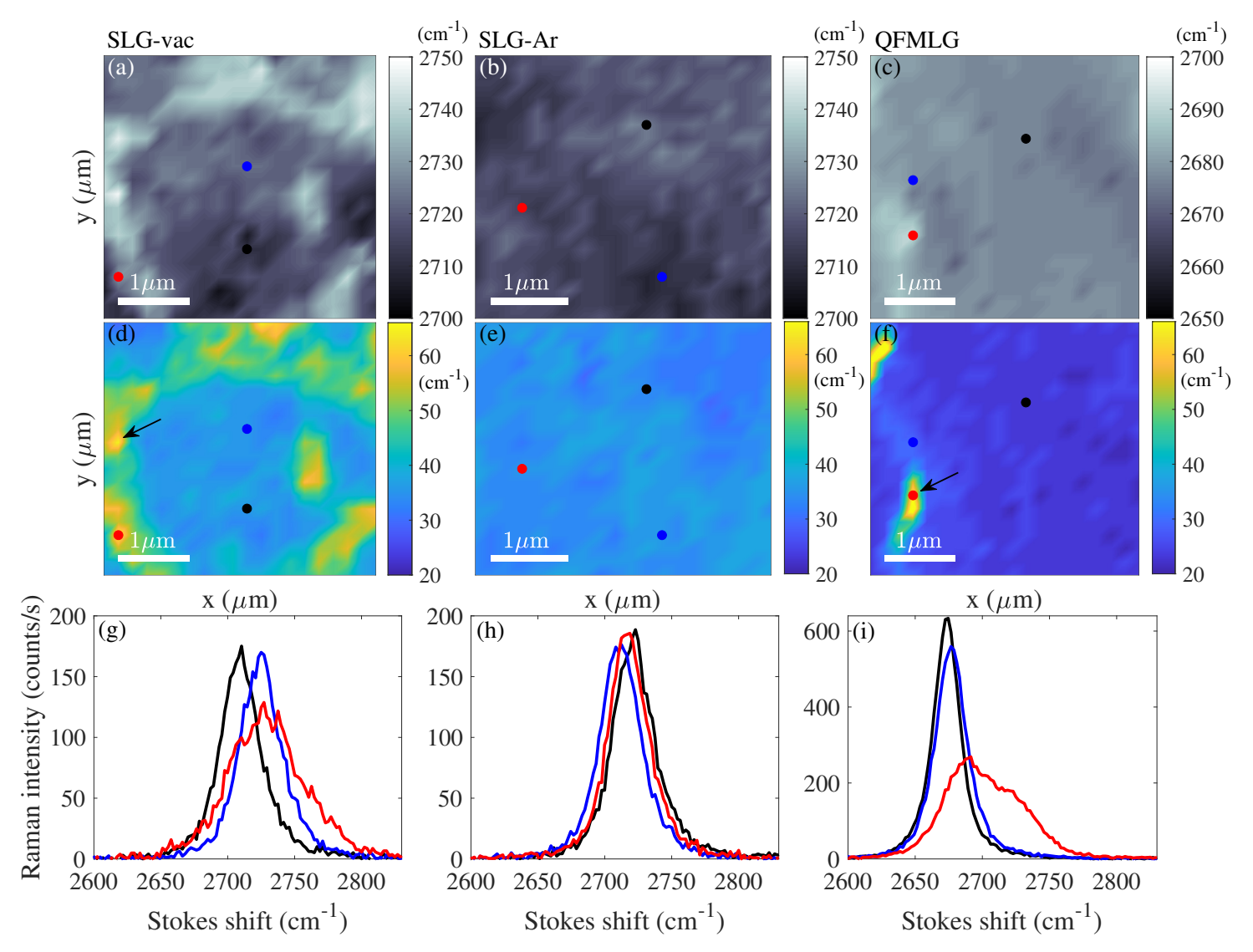

Figure 5. Maps of Raman scattering. The map of (a-c) 2D peak position and (d-f) 2D peak FWHM measured in $(\mathbf{a}, \mathbf{d}, \mathbf{g})$ SLG-vac, $(\mathbf{b}, \mathbf{e}, \mathbf{h})$ SLG-Ar and $(\mathbf{c}, \mathbf{f}, \mathbf{i})$ QFMLG. We also show 2D peak line shape at three locations marked by black, red and blue circles. The spectra are plotted using corresponding black, red and blue curves in $(\mathbf{g}-\mathbf{i})$.

To identify these different areas in topography, we measured also LFM. The LFM can distinguish different materials if their friction with an AFM tip is different. The LFM images are shown in Figure $6 \mathrm{~d}-\mathrm{f}$. We also plot friction force profiles in Figure $6 \mathrm{~g}-\mathrm{i}$. We observe reduction of the friction force at the edges of homogeneous $\mathrm{SiC}$ areas in SLG-vac. Some edges show similar reduction of the friction force in SLG-Ar, too. In both cases, the friction force is reduced $\approx 2 \times$. Such reduction of the friction force was shown between single and bilayer graphene [43]. The friction force is increased in the step edge areas in QFMLG. The increase of friction force was related to the buffer layer [43], however; we observe increase by only $\approx 2.5 \times$. The expected increase is $\approx 10 \times$ for buffer layer. We note, that the step edge, or, sidewall area does not have the same structure as buffer, as shown in literature [44,45]. 
We interpret the graphene at the $\mathrm{SiC}$ step edges as the sidewall graphene ribbons [46,47]. We also observe in LFM that the friction displays large homogeneous areas, and, it also shows considerable amount of sub-micrometer sized inhomogeneities. These patches of different friction/material can contribute to the overall line shape of the 2D peak, too.
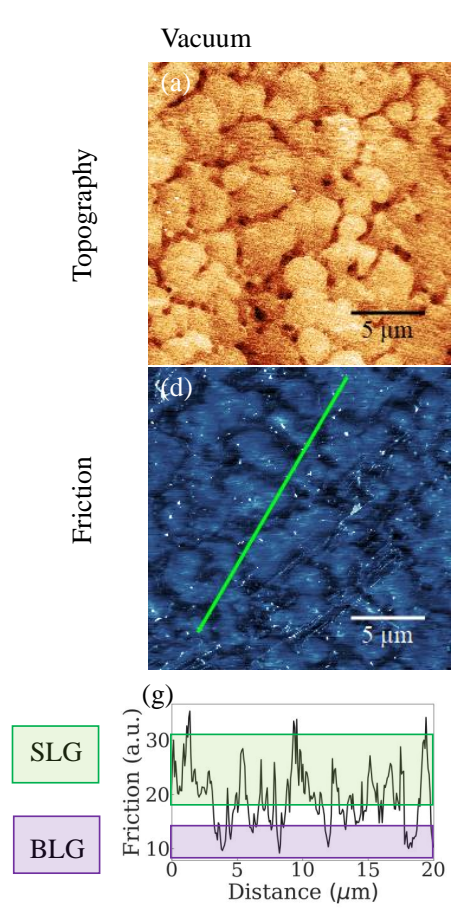
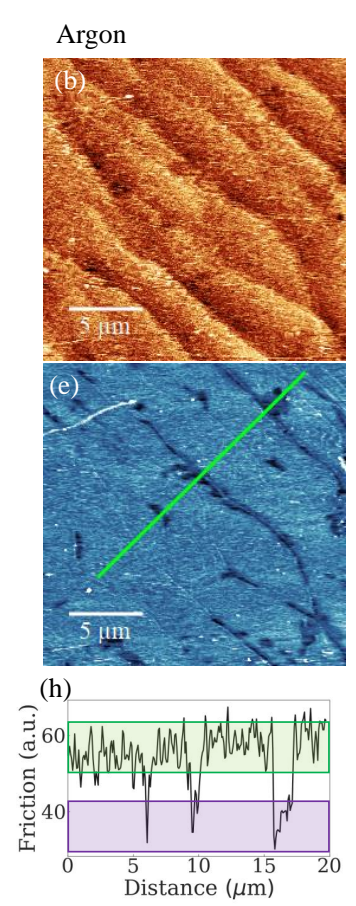
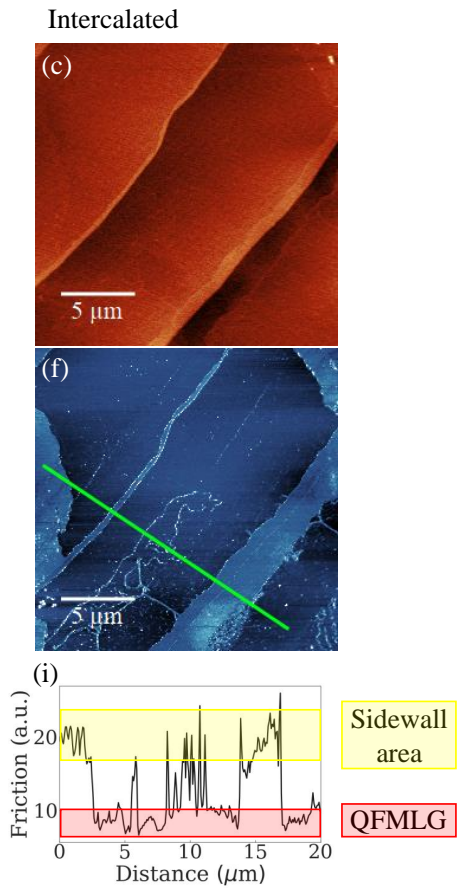

Figure 6. The topographies $\left(20 \times 20 \mu \mathrm{m}^{2}\right)$ of investigated samples are depicted in graphs (a-c) and corresponding friction force maps are shown in graphs $(\mathbf{d}-\mathbf{f})$. The profiles $(\mathbf{g}-\mathbf{i})$ of friction force maps (green lines) demonstrate the SLG, BLG, QFMLG and sidewall area.

Another contribution to the 2D peak broadening is the charge density and strain variation on the sub-micrometer length scale. We analyzed the positions of the $\mathrm{G}$ and 2D peaks measured by Raman mapping. The correlation of the $G$ and 2D peak positions are plotted in the inset of Figure 7 . We describe the relation between the $G$ and $2 D$ peak position $\omega_{G}, \omega_{2 D}$ and mechanical strain and charge density by the following set of two equations.

$$
\begin{gathered}
\omega_{G}=\omega_{G 0}+\alpha_{G}\left|n_{2 D}\right|-2 \gamma_{G} \omega_{G 0} \epsilon \\
\omega_{2 D}=\omega_{2 D 0}-\alpha_{2 D} n_{2 D}-2 \gamma_{2 D} \omega_{2 D 0} \epsilon
\end{gathered}
$$

where proportionality constants are $\alpha_{G}=6.8 \mathrm{~cm}^{-1} / 10^{13} \mathrm{~cm}^{-2}$ and $\alpha_{2 D}=2.7 \mathrm{~cm}^{-1} / 10^{13} \mathrm{~cm}^{-2}$. The effective Grüneisen parameters are $\gamma_{G}=1.8$ and $\gamma_{2 D}=3.5$. The $G$ and 2D peak positions in the charge neutral unstrained graphene are $\omega_{G 0}=1582 \mathrm{~cm}^{-1}$, and $\omega_{2 D 0}=2680 \mathrm{~cm}^{-1}$. We chose the linear dependence of $G$ and 2D peak position on strain in agreement with previous experimental works studying the $G$ peak $[7,26]$ and $2 D$ peak [24-27]. We chose the functional dependence of the $G$ peak position on the charge density as an approximation to the theoretically predicted dependence [7-9,48]. The expected charge density dependence of $G$ peak position is smoothed at room temperature [48] in comparison to $T=0 \mathrm{~K}$. The measured dependence can be clearly approximated by absolute-value function, when compared to theory [48] and experiment [5,7-9]. Our fitting parameters lead to $2.5 \times$ stronger charge density sensitivity of $G$ peak than the sensitivity of $2 \mathrm{D}$ peak. The absolute values and relative strength of $G$ to $2 D$ peak charge sensitivity is in agreement with previous works $[7,9]$. Other fitting parameters lead to the sensitivity of $G$ peak position to strain $\Delta \omega_{G}=-57 \mathrm{~cm}^{-1} / \%$, 
and, to the sensitivity of 2D peak position to strain $\Delta \omega_{2 D}=-188 \mathrm{~cm}^{-1} / \%$. These G and 2D peak sensitivities are in very good agreement with work of Mohiuddin [26], where authors found the G peak sensitivity to the biaxial strain $\Delta \omega_{G}=-63 \mathrm{~cm}^{-1} / \%$ (page 4 in Ref. [26], 2nd column, 1st paragraph). The 2D peak sensitivity to the biaxial strain was found $\Delta \omega_{G}=-191 \mathrm{~cm}^{-1} / \%$, see Ref. [26], page 5, 1 st column, 4th paragraph. These parameters are also in good agreement with an experimental results of Schmidt [5] $\left(\Delta \omega_{G}=-63 \mathrm{~cm}^{-1} / \%, \Delta \omega_{2 D}=-149 \mathrm{~cm}^{-1} / \%\right)$, and, Density Functional Calculations (DFT) $\Delta \omega_{G}=-58 \mathrm{~cm}^{-1} / \%$ and $\Delta \omega_{2 D}=-144 \mathrm{~cm}^{-1} / \%$, see Ref. [26], page 5, 2nd column.

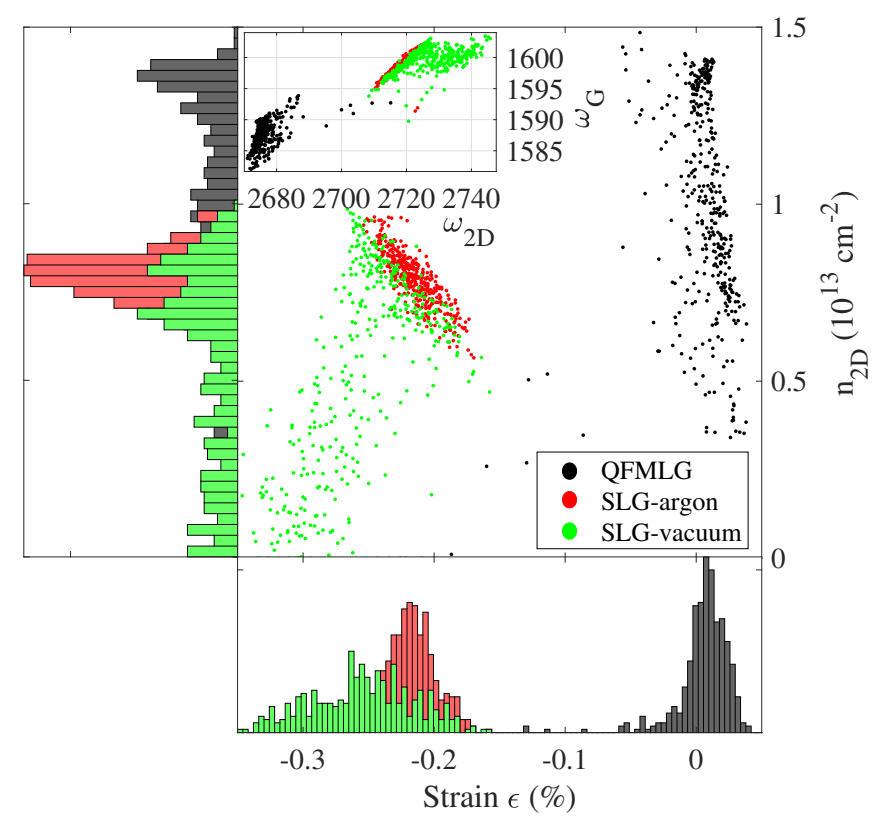

Figure 7. The variation of the charge density and strain as determined from the spectral position of the G and 2D peaks in the three samples (QFMLG, SLG-Ar, SLG-vac). The inset shows the correlation between the $G$ and 2D peak positions. The left and bottom histograms depict the distribution of the charge density and strain, respectively.

We solve the set of Equations (8) and (9) numerically for each point of the Raman map and we obtain the correlation between the charge density and strain, as depicted in Figure 7. We observe that QFMLG shows the largest variation of the charge density. At the same time, the QFMLG sample shows the smallest inhomogeneous broadening, as we showed in spectral analysis of the 2D peak line shape fitted by the Voigt function. The anticorrelation between the observed charge density and inhomogeneous broadening in QFMLG led us to conclude that the main contribution to the sub-micrometer inhomogeneous broadening is the strain variation.

\section{Discussion}

The detailed analysis of 2D peak line shape shows that the Voigt line shape describes the 2D peak better than the Lorentzian line shape. Although the improvement is significant, the $\chi^{2}$ statistics suggests that further corrections are needed. These deviations from the Voigt model are probably due to the larger areas of non-normally distributed strain, or, due to the patches of side wall graphene at the $\mathrm{SiC}$ step edges. Such areas cause side bands or local extrema in the normal distribution of the strain. We propose these deviations from the normal strain distribution can be reduced by defocusing the laser spot. However, defocusing will also reduce the Raman signal, and, it will also cause broader 2D peak. We assumed only biaxial strain contributing the total broadening of 2D peak. Though the uniaxial strain was neglected, it could contribute to the 2D peak broadening, too. The uniaxial strain splits the 2D peak [24-27]. If the 2D peak splitting is smaller or comparable to the spectral resolution $\left(2 \mathrm{~cm}^{-1}\right)$, 
the 2D peak could be misinterpreted as a single-component peak. We assume that the uniaxial strain would effectively contribute to the 2D peak broadening only. Also, since the 2D peak splitting also leads to unequal intensities of the two split components, we expect contribution to the asymmetry of the 2D peak.

Another contribution to the inhomogeneity of epitaxial graphene on $\mathrm{SiC}$ is the stacking alignment between graphene and underlying $\mathrm{SiC}$ substrate [49]. These stacking domains can lead to strain inhomogeneity. However, since the size of these domains is well below the laser spot size $(1 \mu \mathrm{m})$ we assume these stacking domains will mainly contribute to the Gaussian broadening, and, they could be studied by the Voigt line shape fitting.

We note it is necessary to fit the data of the 2D peak in the large enough spectral range. We used a $300 \mathrm{~cm}^{-1}$ broad spectral window. The spectral window broader than $300 \mathrm{~cm}^{-1}$ can not be used due to the presence of the combination Raman mode at $\approx 2450 \mathrm{~cm}^{-1}$. The narrower spectral range can lead to better fit [17,50], however, the fitting parameters might not be as reliable. As the Raman signal is low in the low and high energy tails, the signal might become weaker than the noise level. For the reason of high signal-to-noise ratio the Raman spectra have to be collected with a high numerical aperture objective and the data have to be integrated for at least $60 \mathrm{~s}$.

Our analysis also shows a promise for growth improvements of the epitaxial graphene. The correlation between the width of the 2D peak and carrier mobility has been demonstrated [22]. Hence, we assume, if the strain inhomogeneity is reduced, the graphene carrier mobility could be improved, too.

Contrary to improving graphene, the effect of inhomogeneous broadening can also result in large deviations from the here presented line shapes. We have studied samples where the inhomogeneous broadening is comparable to the homogeneous broadening. However, if the inhomogeneous broadening dominates, the Gaussian component is expected to be prevalent in the 2D peak line shape.

\section{Conclusions}

We show the 2D peak line shape is given by a convolution of the inhomogeneous and homogeneous broadening, so called Voigt broadening, rather than just by a single Lorentzian line shape. We interpreted the inhomogeneous broadening to be mostly given by a sub-micrometer length scale strain variations. The hydrogen intercalated buffer layer is shown to have the smallest homogeneous and inhomogeneous 2D peak broadening.

Author Contributions: Individual contributions of the two authors are following: conceptualization, J.K.; methodology, J.K.; software, J.K. and M.R.; validation, J.K. and M.R.; sample preparation, J.K. and M.R.; formal analysis, J.K. and M.R.; investigation, J.K. and M.R.; resources, J.K.; data curation, J.K. and M.R.; writing-original draft preparation, J.K. and M.R.; visualization, J.K. and M.R.; supervision, J.K.; project administration, J.K.; funding acquisition, J.K. All authors have read and agreed to the published version of the manuscript.

Funding: This research was funded by Czech Science Foundation grant number 19-12052S. The MEYS project VaVpI CZ.1.05/4.1.00/16.0340 is also gratefully acknowledged.

Acknowledgments: We acknowledge helpful comments of J. Bok. These comments led us to the data analysis presented here.

Conflicts of Interest: The authors declare no conflict of interest. The funders had no role in the design of the study; in the collection, analyses, or interpretation of data; in the writing of the manuscript, or in the decision to publish the results.

\section{Abbreviations}

The following abbreviations are used in this manuscript:

SLG single layer graphene

QFMLG Quasi free-standing monolayer graphene

SLG-Ar Single layer graphene grown in argon

SLG-vac Single layer graphene grown in vacuum 


$\begin{array}{ll}\text { AFM } & \text { Atomic Force Microscopy } \\ \text { LFM } & \text { Lateral Force Microscopy } \\ \text { DOF } & \text { Degrees of freedom } \\ \text { RSS } & \text { Residual Sum of Squares } \\ \text { FWHM } & \text { Full Width at Half Maximum } \\ \text { CDF } & \text { Cumulative distribution function }\end{array}$

\section{References}

1. Tang, B.; Guoxin, H.; Gao, H. Raman Spectroscopic Characterization of Graphene. Appl. Spectrosc. Rev. 2010, 45, 369-407. [CrossRef]

2. Ferrari, A.C.; Basko, D.M. Raman spectroscopy as a versatile tool for studying the properties of graphene. Nat. Nanotechnol. 2013, 8, 235-246. [CrossRef] [PubMed]

3. Malard, L.M.; Pimenta, M.A.; Dresselhaus, G.; Dresselhaus, M.S. Raman spectroscopy in graphene. Phys. Rep. 2009, 473, 51-87. [CrossRef]

4. Park, J.S.; Reina, A.; Saito, R.; Kong, J.; Dresselhaus, G.; Dresselhaus, M.S. G ' band Raman spectra of single, double and triple layer graphene. Carbon 2009, 47, 1303-1310. [CrossRef]

5. Schmidt, D.A.; Ohta, T.; Beechem, T.E. Strain and charge carrier coupling in epitaxial graphene. Phys. Rev. B 2011, 84, 235422. [CrossRef]

6. Nicolle, J.; Machon, D.; Poncharal, P.; Pierre-Louis, O.; San-Miguel, A. Pressure-Mediated Doping in Graphene. Nano Lett. 2011, 11, 3564-3568. [CrossRef]

7. Das, A.; Pisana, S.; Chakraborty, B.; Piscanec, S.; Saha, S.K.; Waghmare, U.V.; Novoselov, K.S.; Krishnamurthy, H.R.; Geim, A.K.; Ferrari, A.C.; et al. Monitoring dopants by Raman scattering in an electrochemically top-gated graphene transistor. Nat. Nanotechnol. 2008, 3, 210-215. [CrossRef]

8. Childres, I.; Jauregui, L.A.; Chen, Y.P. Raman spectra and electron-phonon coupling in disordered graphene with gate-tunable doping. J. Appl. Phys. 2014, 116, 233101. [CrossRef]

9. Yan, J.; Zhang, Y.; Kim, P.; Pinczuk, A. Electric field effect tuning of electron-phonon coupling in graphene. Phys. Rev. Lett. 2007, 98, 166802. [CrossRef]

10. Dresselhaus, M.S.; Jorio, A.; Souza Filho, A.G.; Saito, R. Defect characterization in graphene and carbon nanotubes using Raman spectroscopy. Philos. Trans. R. Soc. A Math. Phys. Eng. Sci. 2010, 368, 5355-5377. [CrossRef]

11. Liu, J.; Li, Q.; Zou, Y.; Qian, Q.; Jin, Y.; Li, G.; Jiang, K.; Fan, S. The Dependence of Graphene Raman D-band on Carrier Density. Nano Lett. 2013, 13, 6170-6175. [CrossRef] [PubMed]

12. Maultzsch, J.; Reich, S.; Thomsen, C. Double-resonant Raman scattering in graphite: Interference effects, selection rules, and phonon dispersion. Phys. Rev. B 2004, 70, 155403. [CrossRef]

13. Cancado, L.; Takai, K.; Enoki, T.; Endo, M.; Kim, Y.; Mizusaki, H.; Jorio, A.; Coelho, L.; Magalhaes-Paniago, R.; Pimenta, M. General equation for the determination of the crystallite size L-a of nanographite by Raman spectroscopy. Appl. Phys. Lett. 2006, 88, 163106. [CrossRef]

14. Cancado, L.G.; Jorio, A.; Martins Ferreira, E.H.; Stavale, F.; Achete, C.A.; Capaz, R.B.; Moutinho, M.V.O.; Lombardo, A.; Kulmala, T.S.; Ferrari, A.C. Quantifying Defects in Graphene via Raman Spectroscopy at Different Excitation Energies. Nano Lett. 2011, 11, 3190-3196. [CrossRef]

15. Niyogi, S.; Bekyarova, E.; Itkis, M.E.; Zhang, H.; Shepperd, K.; Hicks, J.; Sprinkle, M.; Berger, C.; Lau, C.N.; de Heer, W.A.; et al. Spectroscopy of Covalently Functionalized Graphene. Nano Lett. 2010, 10, 4061-4066. [CrossRef]

16. Kim, K.; Coh, S.; Tan, L.Z.; Regan, W.; Yuk, J.M.; Chatterjee, E.; Crommie, M.F.; Cohen, M.L.; Louie, S.G.; Zettl, A. Raman Spectroscopy Study of Rotated Double-Layer Graphene: Misorientation-Angle Dependence of Electronic Structure. Phys. Rev. Lett. 2012, 108, 246103. [CrossRef]

17. Kunc, J.; Rejhon, M.; Hlidek, P. Hydrogen intercalation of epitaxial graphene and buffer layer probed by mid-infrared absorption and Raman spectroscopy. AIP Adv. 2018, 8, 045015. [CrossRef]

18. Rejhon, M.; Kunc, J. ZO phonon of a buffer layer and Raman mapping of hydrogenated buffer on SiC(0001). J. Raman Spectrosc. 2019, 50, 465-473. [CrossRef]

19. Basko, D.M. Calculation of the Raman G peak intensity in monolayer graphene: role of Ward identities. New J. Phys. 2009, 11, 095011. [CrossRef] 
20. Ferrari, A.C. Raman spectroscopy of graphene and graphite: Disorder, electron-phonon coupling, doping and nonadiabatic effects. Solid State Commun. 2007, 143, 47-57. [CrossRef]

21. Ferrari, A.C.; Meyer, J.C.; Scardaci, V.; Casiraghi, C.; Lazzeri, M.; Mauri, F.; Piscanec, S.; Jiang, D.; Novoselov, K.S.; Roth, S.; et al. Raman spectrum of graphene and graphene layers. Phys. Rev. Lett. 2006, 97, 187401. [CrossRef] [PubMed]

22. Robinson, J.A.; Wetherington, M.; Tedesco, J.L.; Campbell, P.M.; Weng, X.; Stitt, J.; Fanton, M.A.; Frantz, E.; Snyder, D.; VanMil, B.L.; et al. Correlating Raman Spectral Signatures with Carrier Mobility in Epitaxial Graphene: A Guide to Achieving High Mobility on the Wafer Scale. Nano Lett. 2009, 9, 2873-2876. [CrossRef] [PubMed]

23. Palmer, J.; Kunc, J.; Hu, Y.; Hankinson, J.; Guo, Z.; Berger, C.; de Heer, W.A. Controlled epitaxial graphene growth within removable amorphous carbon corrals. Appl. Phys. Lett. 2014, 105, 023106. [CrossRef]

24. Frank, O.; Mohr, M.; Maultzsch, J.; Thomsen, C.; Riaz, I.; Jalil, R.; Novoselov, K.S.; Tsoukleri, G.; Parthenios, J.; Papagelis, K.; et al. Raman 2D-Band Splitting in Graphene: Theory and Experiment. ACS Nano 2011, 5, 2231-2239. [CrossRef] [PubMed]

25. Huang, M.; Yan, H.; Heinz, T.F.; Hone, J. Probing Strain-Induced Electronic Structure Change in Graphene by Raman Spectroscopy. Nano Lett. 2010, 10, 4074-4079. [CrossRef]

26. Mohiuddin, T.M.G.; Lombardo, A.; Nair, R.R.; Bonetti, A.; Savini, G.; Jalil, R.; Bonini, N.; Basko, D.M.; Galiotis, C.; Marzari, N.; et al. Uniaxial strain in graphene by Raman spectroscopy: G peak splitting, Gruneisen parameters, and sample orientation. Phys. Rev. B 2009, 79, 205433. [CrossRef]

27. Yoon, D.; Son, Y.W.; Cheong, H. Strain-Dependent Splitting of the Double-Resonance Raman Scattering Band in Graphene. Phys. Rev. Lett. 2011, 106, 155502. [CrossRef]

28. Robinson, J.A.; Puls, C.P.; Staley, N.E.; Stitt, J.P.; Fanton, M.A.; Emtsev, K.V.; Seyller, T.; Liu, Y. Raman Topography and Strain Uniformity of Large-Area Epitaxial Graphene. Nano Lett. 2009, 9, 964-968. [CrossRef]

29. Neumann, C.; Reichardt, S.; Venezuela, P.; Droegeler, M.; Banszerus, L.; Schmitz, M.; Watanabe, K.; Taniguchi, T.; Mauri, F.; Beschoten, B.; et al. Raman spectroscopy as probe of nanometre-scale strain variations in graphene. Nat. Commun. 2015, 6, 8429. [CrossRef]

30. Grodecki, K.; Bozek, R.; Strupinski, W.; Wysmolek, A.; Stepniewski, R.; Baranowski, J.M. Micro-Raman spectroscopy of graphene grown on stepped 4H-SiC (0001) surface. Appl. Phys. Lett. 2012, 100, 261604. [CrossRef]

31. Hass, J.; de Heer, W.A.; Conrad, E.H. The growth and morphology of epitaxial multilayer graphene. J. Phys. Condensed Matter 2008, 20, 323202. [CrossRef]

32. De Heer, W.A.; Berger, C.; Ruan, M.; Sprinkle, M.; Li, X.; Hu, Y.; Zhang, B.; Hankinson, J.; Conrad, E. Large area and structured epitaxial graphene produced by confinement controlled sublimation of silicon carbide. Proc. Natl. Acad. Sci. USA 2011, 108, 16900-16905. [CrossRef] [PubMed]

33. Emtsev, K.V.; Bostwick, A.; Horn, K.; Jobst, J.; Kellogg, G.L.; Ley, L.; McChesney, J.L.; Ohta, T.; Reshanov, S.A.; Roehrl, J.; et al. Towards wafer-size graphene layers by atmospheric pressure graphitization of silicon carbide. Nat. Mater. 2009, 8, 203-207. [CrossRef] [PubMed]

34. Yazdi, G.R.; Iakimov, T.; Yakimova, R. Epitaxial Graphene on SiC: A Review of Growth and Characterization. Crystals 2016, 6, 53. [CrossRef]

35. Riedl, C.; Coletti, C.; Iwasaki, T.; Zakharov, A.A.; Starke, U. Quasi-Free-Standing Epitaxial Graphene on SiC Obtained by Hydrogen Intercalation. Phys. Rev. Lett. 2009, 103, 246804. [CrossRef] [PubMed]

36. Hassan, J.; Winters, M.; Ivanov, I.G.; Habibpour, O.; Zirath, H.; Rorsman, N.; Janzen, E. Quasi-free-standing monolayer and bilayer graphene growth on homoepitaxial on-axis $4 \mathrm{H}-\mathrm{SiC}(0001)$ layers. Carbon 2015, 82, 12-23. [CrossRef]

37. Emtsev, K.V.; Speck, F.; Seyller, T.; Ley, L.; Riley, J.D. Interaction, growth, and ordering of epitaxial graphene on $\mathrm{SiC}\{0001\}$ surfaces: A comparative photoelectron spectroscopy study. Phys. Rev. B 2008, 77, 155303. [CrossRef]

38. Speck, F.; Jobst, J.; Fromm, F.; Ostler, M.; Waldmann, D.; Hundhausen, M.; Weber, H.B.; Seyller, T. The quasi-free-standing nature of graphene on H-saturated SiC(0001). Appl. Phys. Lett. 2011, 99, 122106. [CrossRef]

39. Kunc, J.; Rejhon, M.; Belas, E.; Dedic, V.; Moravec, P.; Franc, J. Effect of Residual Gas Composition on Epitaxial Growth of Graphene on SiC. Phys. Rev. Appl. 2017, 8, 044011. [CrossRef] 
40. Ida, T.; Ando, M.; Toraya, H. Extended pseudo-Voigt function for approximating the Voigt profile. J. Appl. Crystallogr. 2000, 33, 1311-1316. [CrossRef]

41. Thompson, P.; Cox, D.; Hastings, J. Rietveld Refinement of Debye-Scherrer Synchrotron X-ray Data from $\mathrm{Al}_{2} \mathrm{O}_{3}$. J. Appl. Crystallogr. 1987, 20, 79-83. [CrossRef]

42. Fromm, F.; Oliveira, M.H., Jr.; Molina-Sanchez, A.; Hundhausen, M.; Lopes, J.M.J.; Riechert, H.; Wirtz, L.; Seyller, T. Contribution of the buffer layer to the Raman spectrum of epitaxial graphene on SiC(0001). New J. Phys. 2013, 15, 043031. [CrossRef]

43. Filleter, T.; McChesney, J.L.; Bostwick, A.; Rotenberg, E.; Emtsev, K.V.; Seyller, T.; Horn, K.; Bennewitz, R. Friction and Dissipation in Epitaxial Graphene Films. Phys. Rev. Lett. 2009, 102, 086102. [CrossRef] [PubMed]

44. Norimatsu, W.; Kusunoki, M. Formation process of graphene on SiC (0001). Physica E 2010, 42, 691-694. [CrossRef]

45. Berger, C.; Conrad, E.H.; de Heer, W.A. Epigraphene: Epitaxial graphene on silicon carbide. arXiv 2017, arXiv:1704.00374.

46. Sprinkle, M.; Ruan, M.; Hu, Y.; Hankinson, J.; Rubio-Roy, M.; Zhang, B.; Wu, X.; Berger, C.; de Heer, W.A. Scalable templated growth of graphene nanoribbons on SiC. Nat. Nanotechnol. 2010, 5, 727-731. [CrossRef]

47. Baringhaus, J.; Aprojanz, J.; Wiegand, J.; Laube, D.; Halbauer, M.; Huebner, J.; Oestreich, M.; Tegenkamp, C. Growth and characterization of sidewall graphene nanoribbons. Appl. Phys. Lett. 2015, 106, 043109. [CrossRef]

48. Lazzeri, M.; Mauri, F. Nonadiabatic Kohn anomaly in a doped graphene monolayer. Appl. Phys. Lett. 2006, 97, 266407. [CrossRef]

49. De Jong, T.A.; Krasovskii, E.E.; Ott, C.; Tromp, R.M.; van der Molen, S.J.; Jobst, J. Intrinsic stacking domains in graphene on silicon carbide: A pathway for intercalation. Phys. Rev. Mater. 2018, 2, 104005. [CrossRef]

50. Pearce, R.; Tan, X.; Wang, R.; Patel, T.; Gallop, J.; Pollard, A.; Yakimova, R.; Hao, L. Investigations of the effect of $\mathrm{SiC}$ growth face on graphene thickness uniformity and electronic properties. Surf. Topogr. Metrol. Properties 2015, 3, 015001. [CrossRef]

(C) 2020 by the authors. Licensee MDPI, Basel, Switzerland. This article is an open access article distributed under the terms and conditions of the Creative Commons Attribution (CC BY) license (http://creativecommons.org/licenses/by/4.0/). 